\title{
BOUNDS FOR HYPERSPHERES OF PRESCRIBED GAUSSIAN CURVATURE
}

\author{
ANDREJS TREIBERGS
}

\begin{abstract}
Apriori $C^{0}$ bounds are established for solutions of A. D. Alexandrov's problem for prescribing the Gauss curvatures of hypersurfaces in Euclidean space. For smooth hyperspheres, this corresponds to finding apriori oscillation bounds for a certain homothety invariant Monge-Ampère equation on the sphere. Conditions are given which are both necessary and sufficient for the boundedness of the hypersphere. An example is given which shows how far Alexandrov's original condition for the existence of solutions must be strengthened to estimate the bound.
\end{abstract}

Apriori $C^{0}$ estimate for A. D. Alexandrov's problem are established in this work. By a convex hypersurface $M^{n}$ of dimension $n$ we mean the boundary of a convex domain $K$ containing a neighborhood of the origin of Euclidean space $\mathbf{E}^{n+1}$. Suppose $M^{n}$ is given as the graph of the unit sphere $\mathbf{S}^{n}$ about the origin with the radius function $r: \mathbf{S}^{n} \rightarrow \mathbf{R}^{+}$. A coordinate map $R: \mathbf{S}^{n} \rightarrow M^{n}$ is given by $R(x)=r(x) x$. Let $\nu: M^{n} \rightarrow \mathbf{S}^{n}$ be the Gauss or normal mapping. In general $\nu(Y)$ is the set of outward unit perpendiculars of supporting hyperplanes to $M^{n}$ at the point $Y$. For smooth $M$ it is the normal vector. Let $d a$ be the standard measure on $\mathbf{S}^{n}$, and write $|F|$ for the $d a$ measure of a Borel set $F$ in $\mathbf{S}^{n}$. Let $O_{n}=\left|\mathbf{S}^{n}\right|$ and let $d(x, y)$ be the distance function on $\mathbf{S}^{n}$. For a set $F \subset$ $\mathbf{S}^{n}$ let $F_{\alpha}=\left\{x \in \mathbf{X}^{n}: d(x, F) \leq \alpha\right\}$ be the $\alpha$-neighborhood of $F$. For convex hypersurface $M^{n},|\nu(R(F))|$ is a nonnegative, completely additive function on the Borel subsets of $\mathbf{S}^{n}$ ([1], [4]) such that $\left|\nu\left(R\left(\mathbf{S}^{n}\right)\right)\right|=O_{n}$. $|\nu(R(F))|$ is invariant under dilations of $M$. Alexandrov's problem is to reconstruct the hypersurface from this measure (see e.g., [11]).

Theorem 1. A. If $\mu$ is a nonnegative, completely additive function on $\Omega$, the set of Borel subsets of $\mathbf{S}^{n}$, such that $\mu\left(\mathbf{S}^{n}\right)=O_{n}$ and, for some

Received November 27, 1985 and, in revised form, January 26, 1989. The author was partially supported by The Sloan Foundation and National Science Foundation Grant NSF-DMS8700783. 
$0<\alpha<\pi / 2$

$$
\mu(F) \leq\left|F_{\alpha}\right| \text { for all } F \in \Omega,
$$

where $F_{\alpha}$ is the $\alpha$-neighborhood of $F$, then any convex hypersurface $M^{n}$ satisfying

$$
\mu(F)=|\nu(R(F))| \text { for all } F \in \Omega
$$

has the ratio of radii bounded

$$
\rho(M):=\frac{\sup \left\{r(x): x \in \mathbf{S}^{n}\right\}}{\inf \left\{r(x): x \in \mathbf{S}^{n}\right\}} \leq c(\alpha, n)<\infty .
$$

B. Conversely, suppose $M^{n}$ is a convex hypersurface about the origin in Euclidean space $\mathbf{E}^{n+1}$. If the radii of $M^{n}$ are bounded in the sense that $\rho\left(M^{n}\right)<\infty$, then there exists $0<\alpha(\rho)<\pi / 2$ so that

$$
|\nu(R(F))| \leq\left|F_{\alpha}\right| \text { for all } F \in \Omega \text {. }
$$

If $M^{n}$ were $C^{2}$, then

$$
|\nu(R(F))|=\int_{R(F)} \kappa d a,
$$

where $\kappa$ is the Gauss-Kronecker curvature of $M^{n}$. If $\mu$ is given by integrating a function,

$$
\mu(F)=\int_{F} f d a,
$$

then, from the expression for $\kappa$, Theorem 1 gives apriori boundedness of weak solutions (satisfying (2)) of the Monge-Ampère equation (e.g., [9])

$$
\operatorname{det}\left(r^{2} \delta_{i j}+2 r_{i} r_{j}-r r_{i j}\right)=f(x) r^{n-1}\left(r^{2}+|D r|^{2}\right)^{(n+1) / 2} \text {, }
$$

where $r_{i}$ and $r_{i j}$ are the coefficients of the first and second covariant derivatives of $r$ in an orthonormal frame on $\mathbf{S}^{n}$ (see [16]). Solutions of (5) are also invariant under dilations $r \mapsto k r$.

A priori $C^{0}$ bounds for the similar reduced higher mean curvature equations ([3], [5], [6], [10], [12]) all require additional decay assumptions that preclude homothety invariance. Condition (1) is more akin through Steiner's formula (27) to isoperimetric conditions on the solubility of the nonparametric mean curvature equations (e.g. [8]). In a future application of this work we will generalize our study [15] of the homothety invariant reduced mean curvature equation.

The question of existence of surfaces satisfying (2) was settled by A. D. Alexandrov [2], who also showed uniqueness up to dilation for such 
surfaces (e.g. [4, p. 30]). The existence of regular solutions to (5) was shown for $n=2$ by Pogorelov [13, Chapter VIII], and for general $n$ by Oliker [9]. Alexandrov's necessary and sufficient conditions that there exist solutions to $(2)$ is

$$
\mu(F)<O_{n}-\left|F^{*}\right| \quad\left(=\left|F_{\pi / 2}\right|\right) \text { for all convex } F \subset \mathbf{S}^{n},
$$

where $F^{*}$ denotes the dual to $F$. (See $\S 1$. There we check that (1) implies (6).)

Alexandrov's condition does not give an apriori $C^{0}$ bound for convex surfaces. $\rho(M)$ can be made arbitrarily large by choosing, say, $M$ to be a sufficiently eccentric ellipsoid, or by moving $M$ so that the origin is sufficiently close to the boundary, whereas (6) holds for any convex body with zero in the interior. In fact, the condition for boundedness strengthens (6) in two ways. First, inequality (6) must be uniformly strict. Second, the inequality must hold for more than just the convex subsets. To see that this second restriction is necessary, we show in $\S 3$ that there are a $\beta>0$ and a sequence of convex surfaces $M_{i}$ so that for each $i$,

$$
\left|\nu\left(R_{i}(F)\right)\right| \leq O_{n}-\left|F^{*}\right|-\beta \text { for all convex } F \subset \mathbf{S}^{n},
$$

but $\rho\left(M_{i}\right)$ tends to infinity.

In $\S 1$ we list some facts about convex hypersurfaces and prove Theorem 1B. In $\S 2$ we prove Theorem 1A. Making a finite version of Alexandrov's argument, our proof depends on two elementary geometric results (Lemmas 2 and 6) about sets in the sphere which are interesting in their own right.

\section{Preliminaries and the necessity of the condition}

In this section we collect some facts about convex sets. A reference is [4]. If $F \in \Omega$ is any set, let $\operatorname{Cone}(F)=\{t X: X \in F, t \geq 0\}$ be the cone generated by $F$. For any cone $C \subset E^{n+1}$, let $C^{*}=\left\{X \in \mathbf{E}^{n+1}: X \cdot Y \leq 0\right.$ for all $0 \neq Y \in C\}$ be the dual cone. $F^{*}=(\operatorname{Cone} F)^{*} \cap \mathbf{S}^{n}$ is the dual angle. We check the equality in (6).

Lemma 1. Suppose $F \subset \mathbf{S}^{n}$ is a convex set. Then $\left|F_{\pi / 2}\right|=O_{n}-\left|F^{*}\right|$.

Proof. By definition, $\operatorname{int}\left(F^{*}\right) \subset \mathbf{S}^{n}-F_{\pi / 2}$. Since $F^{*}$ is convex [4, p. 25] the result follows from $\left|\partial F^{*}\right|=0$ (e.g. [7, pp. 271, 280]).

Corollary. If $\mu$ satisfies (1) for some $0<\alpha<\pi / 2$, then it satisfies (6) .

By homothetic invariance, we may assume that $M$ satisfies $1 \leq r \leq \rho$, In essence we utilize the fact that the unit ball about the origin $U_{1}(0)$ 
is contained in $K$. Alexandrov's condition depends only merely on the origin being in $K$.

Proof of Theorem 1B. For a convex hypersurface $M^{n}$ about the origin such that $\rho(M)<\infty$ we claim condition (4) holds for $\alpha=\cos ^{-1}\left(\rho^{-1}\right)$ $(<\pi / 2)$. To see this it suffices to show that $\nu(R(F))=\bigcup\{\nu(R(x)): x \in$ $F\} \subset F_{\alpha x}$. Choose $x \in F$ and $z \in \nu(R(x))$. Since $z$ is an outward normal of $K$, we have $z \cdot(R(X)-p) \geq 0$ for all $p \in U_{1}$. By choosing $z=p$ we find $r(x) z \cdot x \geq z \cdot z=1$ so that $d(z, x)=\cos ^{-1}(z \cdot x) \leq$ $\cos ^{-1}\left(\rho^{-1}\right)$. q.e.d.

We define the support function to be used later. Let $K \subset \mathbf{R}^{n}$ be a convex body. Let $\phi \in \mathbf{S}^{n-1} \subset \mathbf{R}^{n}$ denote both a coordinate in the standard unit sphere and a unit vector of $\mathbf{R}^{n}$. The support function $p: \mathbf{S}^{n} \rightarrow \mathbf{R}^{n}$ is the distance of the supporting plane in the $\phi$ direction to the origin,

$$
p(\phi)=\sup \{x \cdot \phi: x \in K\} .
$$

In particular, $|p(\phi)| \leq \operatorname{diam}(K)$, where the diameter of a set is the maximal distance between pairs of points in the set.

\section{Proof of Theorem 1A}

In the first part of the proof we show that there is a direction in which $K$ has linear growth. Let $L_{s, \theta}=\left\{X \in \mathbf{E}^{n+1}: X \cdot \theta=s\right\}$ be the hyperplane in $\mathbf{E}^{n+1}$ normal to $\theta$, and a distance $s$ from the origin. Let $H_{s, \theta}=$ $\bigcup\left\{L_{\sigma, \theta}: \sigma \leq s\right\}$ be a halfspace which it bounds. Let $K_{s, \theta}=L_{s, \theta} \cap K$. We show that for some $\theta_{0}$ the diameter satisfies

$$
\operatorname{diam} K_{s, \theta_{0}} \leq c_{2}+c_{3} s
$$

where $c_{2}$ and $c_{3}$ depend only on $\alpha$ and $n$.

To see this let $\beta=\alpha / 2+\pi / 4$ and let the open geodesic ball $B_{\beta}((-1,-, \cdots, 0)) \subset \mathbf{S}^{n}$. By (1) applied to $\mathbf{S}^{n}-B_{\beta}$ and using [4, p. 26], we obtain

$$
\left.\left|\nu\left(R\left(B_{\beta}\right)\right)\right|=O_{n}-\left|\nu\left(R\left(\mathbf{S}^{n}-B_{\beta}\right)\right)\right| \geq O_{n}-\left|B_{\beta-\alpha,}\right|\right)=c_{4}>0,
$$

where $c_{4}$ depends only on $\alpha$ and $n$. To show that $B_{\beta}$ contains a useful concentration of curvature we apply

Lemma 2. Let $F \subset \mathbf{S}^{n}$ be a Borel set with $|F| \geq \mu>0$. Then there are $n+1$ points $x_{i} \in F$ in an open hemisphere of $\mathbf{S}^{n}$ whose convex hull $T$ contains a geodesic ball $B_{\varepsilon}(\theta)$ with $\varepsilon=c_{5}(n) \mu^{n}>0$.

Proof. Let $B_{\pi / 4}$ be a fixed ball in $\mathbf{S}^{n}$ and $\left\{y_{j}: j=1, \cdots, N\right\}$ be a maximal set of points in $B_{\pi / 4}$ such that $d\left(y_{j}, y_{k}\right) \geq 4 \delta$ for all $j \neq k$ and 
for some sufficiently small $\delta$ to be chosen later. By maximality, $\bigcup_{j} B_{4 \delta}\left(y_{j}\right)$ is a cover of $B_{\pi / 4}$. Hence $N\left|B_{4 \delta}\right| \geq\left|B_{\pi / 4}\right|$ so there is a constant $c_{6}(n)>0$ so that

$$
N \geq c_{6} \delta^{-n} .
$$

Let $C=\bigcup_{j} B_{\delta}\left(y_{j}\right)$. Let $G$ be the group of isometries of $\mathbf{S}^{n}$, and $d G$ the invariant unit measure on $G$. By Brother's Poincaré formula [14, p. 277] there is a $c_{7}(n)>0$ such that

$$
\int|F \cap g C| d G=c_{7} N|F|\left|B_{\delta}\right| .
$$

Hence, for some motion, say $g=\mathrm{id}$, by $(10)$

$$
|F \cap C| \geq c_{8} \mu,
$$

where $c_{8}$ depends only on $n$. Thus there are at least $k$ balls $\left\{B_{\delta}\left(y_{j^{\prime}}\right)\right\}$ so that $\left|B_{\delta}\left(y_{j^{\prime}}\right) \cap F\right|>0$, where $k\left|B_{\delta}\right| \geq c_{8} \mu$. On the other hand not all $B_{\delta}\left(y_{j^{\prime}}\right)$ lie in a $3 \delta$ neighborhood of any great $\mathbf{S}^{n-1}$ in $B_{\pi / 4}$ because otherwise

$$
c_{8} \mu \leq k\left|B_{\delta}\right| \leq 6 \delta O_{n-1},
$$

which is a contradiction for the choice $\delta=.1 c_{8} \mu / O_{n-1}$.

By construction there are points $x_{j^{\prime}} \in B_{\delta}\left(y_{j^{\prime}}\right) \cap F$ which are pairwise $2 \delta$ apart. Now we show the existence of the desired $n+1$ points by iterating $m$, the number of points chosen. For $m=2$ take any two $x_{j^{\prime}}$ 's. We may put $\varepsilon_{1}=\delta$. Suppose $m$ points have been chosen which lie in some great $\mathbf{S}^{m-1}$ and whose convex hull contains a $B_{m-1}^{\prime}=B_{\varepsilon_{m-1}}\left(\theta_{m-1}\right) \cap \mathbf{S}^{m-1}$ for some $\theta_{m-1} \in \mathbf{S}^{m-1}$. Not all $B_{\delta}\left(y_{j^{\prime}}\right)$ 's lie in a $3 \delta$ neighborhood of this $\mathbf{S}^{m-1}$. Hence there is an $x_{m}$ in such an outlying ball so that $d\left(\mathbf{S}^{m-1}, x_{m}\right)>\delta$. Let $\gamma$ denote the geodesic segment from $\theta_{m-1}$ to $x_{m}$, and $q$ any point of $\mathbf{S}^{m-1}-\left\{\theta_{m-1}\right\}$. Let $\theta_{m}$ be the midpoint of $\gamma$ and $\sin \varepsilon_{m}=.5 \sin \delta \sin \varepsilon_{m-1}$. Then the ball $B_{m}^{\prime}=B_{\varepsilon_{m}}\left(\theta_{m}\right) \cap \mathbf{S}^{m}$ is contained in the convex hull of $B_{m-1}^{\prime}$ and $x_{m}$, where $\mathbf{S}^{m}$ is the great sphere containing $\mathbf{S}^{m-1}$ and $x_{m}$. Let $q^{\prime}$ be the point on the geodesic extending the ray $\theta_{m-1} q$ closest to $x_{m}$. Let $a$ be the angle from $\gamma$ to the ray. By the law of sines restricted to the great $\mathbf{S}^{2}$ containing the ray and the segment,

$$
\sin a \geq \sin a \sin d\left(x_{m}, \theta_{m-1}\right)=\sin d\left(x_{m}, q^{\prime}\right) \geq \sin \delta .
$$


We show that $B_{m}^{\prime} \cap \mathbf{S}^{m-1}$ is empty. To see this, let $q \in \mathbf{S}^{m-1}$ be the closest point to $\theta_{m}$. If $q=\theta_{m-1}$, then, by the definition of $\varepsilon_{m}, d\left(q, \theta_{m}\right) \geq \varepsilon_{m}$. Otherwise, as before,

$$
\begin{aligned}
\sin d\left(\theta_{m}, q\right) & =\sin a \sin d\left(\theta_{m-1}, \theta_{m}\right) \\
& \geq \frac{1}{2} \sin ^{2} \delta \geq \frac{1}{2} \sin \delta \sin \varepsilon_{m-1},
\end{aligned}
$$

and apply the triangle inequality. To see that $B_{m}^{\prime}$ is in the convex hull of $B_{m-1}^{\prime}$ and $x_{m}$, assume to the contrary that there is a geodesic $\zeta$ through $x_{m}$ which meets $B_{m}^{\prime}$ but not $B_{m-1}^{\prime}$. Let $y \in \zeta \cap B_{m}^{\prime}$ be the closest point to $\theta_{m}$ and let $\phi$ be the angle from $\zeta$ to $\gamma$. By the law of sines for the triangle $y x_{m} \theta_{m}$,

$$
\frac{1}{2} \sin \phi \sin d\left(x_{m}, \theta_{m-1}\right) \leq \sin \phi \sin d\left(\theta_{m}, x_{m}\right) \leq \sin \varepsilon_{m} .
$$

On the other hand, letting $q=\zeta \cap \mathbf{S}^{m-1} \notin B_{m-1}^{\prime}$ and $b$ be the angle $x_{m} q \theta_{m-1}$ and noting the symmetry of the $a$ estimate in $q$ and $\theta_{m-1}$ yields

$$
\sin \delta \sin \varepsilon_{m-1} \leq \sin b \sin \varepsilon_{m-1}<\sin d\left(\theta_{m-1}, x_{m}\right) \sin \phi,
$$

a contradiction. When $m=n$ the iteration stops with $\theta=\theta_{n}$ and $\varepsilon=\varepsilon_{n}$. q.e.d.

To resume the proof of Theorem 1A, apply Lemma 2 to the set $\nu\left(R\left(B_{\beta}\right)\right)$ $\subset \mathbf{S}^{n}$ with measure at least $c_{4}$ to show that it contains $n+1$ points $z_{i}$ in $\nu\left(R\left(B_{\beta}\right)\right)$ whose convex hull contains a ball $B_{\varepsilon}(-\theta)$, where $\varepsilon$ is positive and depends on $c_{4}$ and $n . K$ is contained in the supporting half-spaces, i.e.,

$$
K \subset \bigcap_{i=0}^{n} H_{r\left(x_{i}\right), z_{i}} \subset U_{c_{2}}+\operatorname{Cone}(T)^{*} \subset U_{c_{2}}+\operatorname{Cone}\left(B_{\varepsilon}(-\theta)\right)^{*},
$$

where + is Minkowski addition of sets, and $\sup \left\{r\left(x_{i}\right): i=1, \cdots\right.$, $n-1\} \leq c_{2} \tan \beta$, since all the supporting planes intersect $\operatorname{Cone}\left(B_{\beta}\right) \cap$ $H_{1,(-1,0, \cdots, 0)}$. Thus in the $\theta$ direction, $K$ has linear growth bounded by $c_{2}$ and $c_{3}=\cot (\varepsilon)$. In particular $\operatorname{diam}\left(K_{1}, \theta\right) \leq c_{9}=c_{2}+c_{3}$.

In the second part of the proof, after rotating the coordinate axes so that $\theta$ is in the $x_{1}$ direction, we show that $K$ has finite extent in the $x_{1}$ direction. For simplicity, denote $L_{s}=L_{s, \theta}, K_{s}=L_{s} \cap K$ and $H_{s}=H_{s, \theta}$. Since $K-H_{s}$ is convex, $F_{x}=R^{-1}\left(K_{s}\right)$ is convex in $\mathbf{S}^{n}$. Applying (1) we find

$$
\left|\nu\left(R\left(F_{s}\right)\right)\right| \leq\left|\left(F_{s}\right)_{\alpha}\right|
$$


To go further, let $p(s, \phi)$ be the support function for $K_{s}$ in $L_{s}$ as in (8). If we denote the spherical coordinate $\phi \in \mathbf{S}^{n-1} \subset L_{s}$, then $\{X \in$ $\left.L_{s}: X \cdot \phi=p(s, \phi)\right\}$ is the supporting plane to $K_{s}$ with outward normal vector $\phi$. Consider the convex hull $W_{s, t}$ of $K_{s}$ and $K_{t}$ in $\mathbf{E}^{n+1}$ for $s<t$. We wish to compute the total curvature of the right half of $W_{s, t}$, namely, $Q=\partial W_{s, t}-H_{(s+t) / 2}$. In order to do this, note that given $\phi$, the supporting plane to $\partial Q$ has a normal vector which defines the angle $a(\phi)$,

$$
n=\frac{\phi-\theta \Delta}{\sqrt{1+\Delta^{2}}}=\phi \sin a+\theta \cos a,
$$

where $\Delta=(p(s, \phi)-p(t, \phi)) /(s-t)$. If $\xi$ denotes the Gauss map for $\partial W_{s, t}$,

$$
\begin{aligned}
|\xi(Q)| & =\left|\left\{\exp _{\theta}(s \phi): 0 \leq s \leq a(\phi), \phi \in \mathbf{S}^{n-1}\right\}\right| \\
& =\int_{\mathbf{S}^{n-1}} \int_{0}^{a(\phi)} \sin ^{n-1} s d s d \phi,
\end{aligned}
$$

where we have used $(s, \phi)$ as polar coordinates for $\mathbf{S}^{n}$ about $\theta$ and $\exp _{\theta}$ as the exponential map from the tangent space to $\mathbf{S}^{n}$. We substitute $u=\cos s$ to find

$$
|\xi(Q)|=\frac{1}{2} O_{n}-\int_{\mathbf{S}^{n-1}} f_{n}(\cos a) d \phi,
$$

where

$$
f_{n}(v)=\int_{0}^{v}\left(1-u^{2}\right)^{(n-2) / 2} d u
$$

By convexity and using hypothesis (1), for any $\alpha \leq \beta<\pi / 2$ we have

$$
|\xi(Q)| \leq\left|\nu\left(M-H_{s}\right)\right| \leq\left|\left(R^{-1}\left(M-H_{s}\right)\right)_{\beta}\right|,
$$

which may be computed like $|\xi(Q)|$ by viewing $s=0$ and " $p(0, \phi)=0$ ". Hence

$$
\left|\left(R^{-1}\left(M-H_{S}\right)\right)_{\beta}\right|=\frac{1}{2} O_{n}-\int_{\mathbf{S}^{n-1}} f_{n}(\cos (a+\beta-\pi / 2)) d \phi,
$$

since $F_{s}$ is convex on $\mathbf{S}^{n}$. Now substituting (11), (12), and (14) in (13) and letting $s \rightarrow t-$, we obtain

$$
\int_{\mathbf{S}^{n-1}} f_{n}\left(g\left(p_{s}\right)\right) d \phi \leq \int_{\mathbf{S}^{n-1}} f_{n}\left(\sin \beta g\left(\frac{p}{s}\right)-\cos \beta \sqrt{1-g^{2}\left(\frac{p}{s}\right)}\right) d \phi,
$$

where $p_{s}$ denotes the left derivate of $p(s, \phi)$ and

$$
g(u)=\frac{u}{\sqrt{1+u^{2}}} .
$$


By (9), $u=p / s$ satisfies $|u| \leq c_{10}=\max \left\{c_{9}, \tan \alpha\right\}$ whenever $s \geq 1$. We may simplify the right side by a pointwise inequality.

Lemma 3. For $m>0$ there is a $\gamma(m), 0<\gamma<\pi / 2$, so that if $|u| \leq m$, then

$$
(\sin \gamma) g(u)-\cos \gamma \sqrt{1-g^{2}(u)} \leq g\left(\left(1-\varepsilon_{1}\right) u-\varepsilon_{2}\right),
$$

for $g$ given by (16) and for any constants $1-\varepsilon_{1} \geq \sin \gamma$ and $\varepsilon_{2} \leq \cos ^{2} \gamma$.

Proof. Define $0<\gamma<\pi / 2$ by $g(m)=\sin \gamma$. For $u \geq 0$, by concavity and $\operatorname{Lip}(g)<1$, we have

$$
\begin{aligned}
h_{\gamma}(u) & =(\sin \gamma) g(u)-\cos \gamma \sqrt{1-g^{2}(u)} \\
& \leq g(u \sin \gamma)-\cos ^{2} \gamma \leq g\left(\left(1-\varepsilon_{1}\right) u-\varepsilon_{2}\right) .
\end{aligned}
$$

If $u \leq 0$, then by writing $g(u)=-\sin z, 0 \leq z \leq \gamma, h_{\gamma}=-\cos (\gamma-z)$ and the result follows from the trigonometric inequality

$$
g^{-1}\left(h_{\gamma}\right)=-\cot (\gamma-z) \leq-\tan z-\cot \gamma \leq\left(1-\varepsilon_{1}\right) u-\varepsilon_{2} \text {. q.e.d. }
$$

By Lemma 3 there is a $\gamma\left(c_{10}(\alpha, n)\right) \geq \alpha$ so that (15) becomes

$$
\int_{\mathbf{S}^{n-1}} f_{n} \circ g\left(p_{s}\right) \leq \int_{\mathbf{S}^{n-1}} f_{n} \circ g\left(\left(1-\varepsilon_{1}\right) \frac{p}{s}-\varepsilon_{2}\right)
$$

for any $1-\varepsilon_{1} \geq \sin \gamma$ and $\varepsilon_{2} \leq \cos ^{2} \gamma$.

We now show $K_{s}=\varnothing$ for some $s<s_{1}$, with $s_{1}$ large and depending only on $n$ and $\alpha$ (to be described). Suppose this were not the case. Then $M^{n}$ must contain a long midsection in which it is nearly conical. This is first shown in an $L^{1}$ sense.

Lemma 4. Let $p(s, \phi)$ be the support function for the sections $K_{s}$ of a convex body $0 \in K$ as in $(8)$, with $p(1, \phi) \leq c_{9}$. Let

$$
P(x)=\int_{\mathbf{S}^{n-1}} \frac{p(s, \phi)}{s} d \phi .
$$

Then for all $\delta>0$ there exists $s_{1}\left(\delta, c_{9}\right)$ so that if $P(s)>0$ for $1 \leq s \leq s_{1}$, then for some $s_{0}, 1 \leq s_{0} \leq 2 s_{0} \leq s_{1}$,

$$
(1-\delta) P\left(s_{0}\right) \leq P\left(2 s_{0}\right) \text {. }
$$

Proof. First observe $p_{s} \leq p / s$. Fixing $\phi \in \mathbf{S}^{n-1}$, consider the orthogonal projection $K^{\prime}$ of $K$ onto the $(\theta, \phi)$ plane. Since $K^{\prime}$ is still convex and $p(s, \phi)$ is the distance from $s \theta$ to $\partial K^{\prime}$ in the $\phi$ direction, we see that rays from the origin pierce outwards any secant of $\partial K^{\prime},(s, p(s, \phi))$ to $(t, p(t, \phi)), s<t$. Hence in the notation of $(11), \Delta \leq p / s$ so $P_{s} \leq 0$. 
Changing to $s=2^{t}$ and $u=\log P$ we still have $u_{t} \leq 0$. If $P(t)>0$ for $0 \leq t \leq 2 T+2$ then we have $P(s) \geq 1 / 2$ for $s \leq T+1$, since $P(s)$ is independent of the choice of origin of $L_{s}$, and $K$ contains the convex hull of $B_{1}(0)$ and a point in $K_{2 T+2}$. But $P(s)$ is absolutely continuous, so

$\log u(0)+\log 2 \geq u(0)-u(T+1)=-\int_{0}^{T+1} u_{t}(x) d x \geq-\sum_{i=0}^{[T]} \int_{i}^{i+1} u_{t}(x) d x$,

where $[T]$ is the greatest integer function. Hence for some $i^{\prime}$,

$$
u\left(i^{\prime}\right)-u\left(i^{\prime}+1\right) \leq \frac{\log c_{9}+\log O_{n-1}+\log 2}{T} .
$$

The result follows if we set $s_{1}=4\left(2 c_{9} O_{n-1}\right)^{2 / \delta}$. q.e.d.

Next we show that a long body $K$ is nearly conical at $K_{s_{0}}$ in measure. In $L_{s_{0}}$, by convexity, the two convex sets satisfy $K_{1}=\frac{1}{2} K_{2 s_{0}} \subset K_{2}=K_{s_{0}}$ where we mean the Minkowski multiplication of sets in $\mathbf{E}^{n+1}$. The support functions $q_{i}$ for $K_{i}$ satisfy

$$
q_{1}(\phi)=\frac{1}{2} p\left(2 s_{0}, \phi\right) \leq q_{2}(\phi)=p\left(s_{0}, \phi\right) .
$$

By (9), $\left|q_{i}\right| \leq s_{0} c_{9}$. We need to deduce from (18) that the support functions are close in measure.

Lemma 5. Let $K_{1} \subset K_{2} \subset B_{m}(0) \subset \mathbf{R}^{n}$ be two convex sets. For all $\varepsilon>0$ there is a $\delta(\varepsilon, n)>0$ so that if

$$
\int_{\mathbf{S}^{n-1}} q_{1}(\phi) d \phi \geq(1-\delta) \int_{\mathbf{S}^{n-1}} q_{2}(\phi) d \phi
$$

for the support functions $q_{i}$ of $K_{i}$ as in (8), then there is a set $F \subset \mathbf{S}^{n-1}$, $|F| \leq \varepsilon$, such that for all $\phi \in \mathbf{S}^{n-1}-F$,

$$
q_{1}(\phi) \geq(1-\varepsilon) q_{2}(\phi)-2 m \varepsilon .
$$

Proof. Let $F=\left\{\phi \in \mathbf{S}^{n-1}: q_{1}(q)<(1-\varepsilon) q_{2}(\phi)-2 m \varepsilon\right\}$. Then

$$
\delta O_{n-1} m \geq \delta \int q_{2} \geq \int q_{2}-q_{1} \geq \varepsilon \int_{F} 2 m+q_{2} \geq|F| \varepsilon m .
$$

The lemma follows by choosing $\delta=\varepsilon^{2} / O_{n-1}$. q.e.d.

Thus there is a set $F \subset \mathbf{S}^{n-1},|F| \leq \varepsilon$ and such that

$$
\frac{1}{2} p\left(2 s_{0}, \phi\right) \geq(1-\varepsilon) p\left(s_{0}, \phi\right)-2 \varepsilon c_{9} s_{0}
$$


for all $\phi \in \mathbf{S}^{n-1}-F$ and any $\varepsilon$ with corresponding $\delta$ and $s_{1}$. Hence for $\phi \notin F$, by convexity and (19) we may estimate

$$
p_{s}\left(s_{0}, \phi\right) \geq \frac{p\left(2 s_{0}, \phi\right)-p\left(s_{0}, \phi\right)}{s_{0}} \geq(1-2 \varepsilon) \frac{p\left(s_{0}, \phi\right)}{s_{0}}-4 \varepsilon c_{9} .
$$

Finally, there is a positive lower bound

$$
\frac{d}{d u} f_{n}(g(u)) \geq c_{11}(\alpha, n)>0
$$

for $|u| \leq c_{9}+1$. Since $|p / s| \leq c_{9}$ and $\left|f_{n} \circ g\right| \leq 1$,

$$
\begin{aligned}
\int_{\mathbf{S}^{n-1}} f_{n} \circ g\left(p_{s}\right) \geq & \int_{\mathbf{S}^{n-1}-F} f_{n} \circ g\left((1-2 \varepsilon) \frac{p}{s}-4 \varepsilon c_{9}\right)-2|F| \\
\geq & \int_{\mathbf{S}^{n-1}-F} f_{n} \circ g\left((1-2 \varepsilon) \frac{p}{s}-4 \varepsilon c_{9}-\frac{\varepsilon^{1 / 2}}{c_{11}}\right) \\
& -2 \varepsilon+\left(O_{n-1}-\varepsilon\right) \varepsilon^{1 / 2} \\
\geq & \int_{\mathbf{S}^{n-1}} f_{n} \circ g\left((1-2 \varepsilon) \frac{p}{s}-4 \varepsilon c_{9}-\frac{\varepsilon^{1 / 2}}{c_{11}}\right) \\
& -4 \varepsilon+\left(O_{n-1}-\varepsilon\right) \varepsilon^{1 / 2},
\end{aligned}
$$

provided that $4 \varepsilon c_{9}+\varepsilon^{1 / 2} / c_{11} \leq 1$. Now choosing $\varepsilon$ depending only on $\alpha$ and $n$ so small that $1-2 \varepsilon \geq \sin \gamma, 4 \varepsilon c_{9}+\varepsilon^{1 / 2} / c_{11} \leq \cos ^{2} \gamma$ and $4 \varepsilon<\left(O_{n-1}-\varepsilon\right) \varepsilon^{1 / 2}$, we get a contradiction to (17).

\section{A counterexample to boundedness}

The estimate of the bound in Theorem 1 requires that Alexandrov's condition be strengthened in two ways, by making the inequality uniformly strict and by requiring that the condition hold for more than the convex sets. In this section we given an example which shows that making the inequality in Alexandrov's condition strict by itself does not provide an apriori $C^{0}$ bound. We begin with an estimate of the width of a convex set in $\mathbf{S}^{2}$ in terms of its area.

Lemma 6. Suppose $F \subset \mathbf{S}^{2}$ is a convex set. Let $D$ be the diameter of $F$. Then there exist $v_{1}, v_{2}$ in $\mathbf{S}^{2}, d\left(v_{1}, v_{2}\right)=\lambda$, so that the lune

$$
V_{\lambda}=\left\{x \in \mathbf{S}^{2}: x \cdot v_{1} \leq 0, x \cdot v_{2} \geq 0\right\} \supset F
$$

provided that

$$
|F| \leq \lambda \tan \frac{1}{4} D
$$


or

$$
16 \pi|F| \leq \lambda^{2}
$$

Proof. Choose $x, y$ in the closure of $F$, which realize the diameter. Extend the geodesic $\gamma$ from $x$ to $y$ to a semi-great circle exyf centered at the midpoint of $\gamma$. Consider the smallest lune $V_{\lambda}$ with vertices $e$, $f$ containing $F$. Let $V_{\lambda^{\prime}}$ be one of the components of $V_{\lambda}-\gamma$, and $z$ a point of contact between $\partial V_{\lambda^{\prime}}$ and $F$ not on $\gamma . V_{\lambda^{\prime}}$ is divided into three disjoint geodesic triangles $T=x y z, T_{1}=e x z$ and $T_{2}=f y z$. Let the lengths $d^{\prime}=d(e, x), d_{1}=d(e, z)$ and $d_{2}=d(z, f)$. We have $2 d^{\prime}+D=d_{1}+d_{2}=\pi$. By the triangle inequality $d_{i} \geq d^{\prime}$ since $\pi-d_{i}=d_{3-i} \leq d^{\prime}+D=\pi-d^{\prime}$. To estimate the area of $T$ in terms of $\lambda^{\prime}$ we appy the area formula for spherical triangles to find

$$
\sin \lambda^{\prime} \cot \frac{1}{2}\left|T_{i}\right|=\cot \frac{1}{2} d^{\prime} \cot \frac{1}{2} d_{i}+\cos \lambda^{\prime} \text {. }
$$

Supposing $d_{1} \leq d_{2} \leq \pi-d^{\prime}$ we find using (23) that $\left|T_{2}\right| \leq \lambda^{\prime}$. Hence we may estimate

(24) $|T|=\left|V_{\lambda^{\prime}}\right|-\left|T_{1}\right|-\left|T_{2}\right| \geq \lambda^{\prime}-2 \cot ^{-1}\left(\cot \frac{1}{2} d^{\prime} \cot \frac{1}{2} d_{1} \csc \lambda^{\prime}+\cot \lambda^{\prime}\right)$.

For $\varepsilon, \lambda>0$, using the concavity of $\sin [(1+\varepsilon) \lambda /(2+\varepsilon)]$ and calculus one finds

$$
\frac{1+\varepsilon+\cos \lambda}{\sin \lambda}>\cot \left(\frac{\lambda}{2+\varepsilon}\right) \text {. }
$$

Applying this with $1+\varepsilon=\cot \frac{1}{2} d^{\prime} \cot \frac{1}{2} d_{1}$ to (24) and using $d^{\prime}=(\pi-D) / 2$ and $d_{1} \leq \pi / 2$ yield (21) after summing both sublunes of $V_{\lambda}$. Since some ball $B_{D} \supset F$ we have

$$
|F| \leq\left|B_{D}\right|=4 \pi \sin ^{2} \frac{1}{2} D \text {. }
$$

Combining this with $\tan \frac{1}{4} D \geq \frac{1}{2} \sin \frac{1}{2} D$ in (21) gives (22).

Theorem 2. There are a positive $\beta$ and a sequence of convex two-dimensional surfaces $M_{i}$ about the origin for which the bound on the ratio of radii (3) satisfies $\rho\left(M_{i}\right) \rightarrow \infty$ as $i \rightarrow \infty$ but for all $i$,

$$
\left|\nu_{i}\left(R_{i}(F)\right)\right|<O_{2}-\left|F^{*}\right|-\beta \text { for all convex } F \subset \mathbf{S}^{2} .
$$

Proof. We show the condition is satisfied by surfaces of rotation $M_{i}$ given for each positive integer $i$ by rotating about the $x$-axis:

$$
\begin{array}{ll}
x=-1 & \text { if } 0 \leq y \leq i \text { and } x=-1, \\
y=i+\sqrt{1-x^{2}} & \text { if }-1<x \leq 0 \\
y=\sqrt{(i+1)^{2}-x^{2}} & \text { if } 0<x \leq i+1 .
\end{array}
$$


For these surfaces, $\rho\left(M_{i}\right)=i+1$. In general, the same argument will show that if $M$ is any convex hypersurface which has a planar neighborhood about some $Y \in M$, then $M_{i}=M-X_{i}$, where $X_{i}$ is a sequence tending to $Y$ in $\mathbf{E}^{n+1}$, also satisfies (7) but $\rho_{i} \rightarrow \infty$. The differentiability provides the convenience of an absolutely continuous Gauss map so $\left|\nu_{i}\left(R_{i}(\partial F)\right)\right|=$ 0 . For convex $F \in \mathbf{S}^{2}$ we have by Lemma 1 ,

$$
\left|F^{*}\right|=O_{2}-\left|F_{\pi / 2}\right| \text {. }
$$

Next, by Steiner's formula for space forms [14, p. 322],

$$
\left|F_{\pi / 2}\right|=l(\partial F)+2 \pi,
$$

where $l$ is the length. The isoperimetric inequality on spheres [14, p. 324] is

$$
l(\partial F)^{2} \geq 4 \pi|F|-|F|^{2} .
$$

We shall establish for some $\beta>0$, but independent of $i$, and for $J(u)=$ $\sqrt{4 \pi u-u^{2}}$

$$
\begin{aligned}
I(F) & =\left|\nu\left(R_{i}(F)\right)\right|\left(=\int_{R_{i}(F)} \kappa_{i} d a_{i}\right) \\
& \leq 2 \pi+J(|F|)-\beta \quad \text { for all convex } F \in \mathbf{S}^{2},
\end{aligned}
$$

which by (26), (27) and (28) is sufficient for (25).

We decompose sets into the disjoint union $F=F^{-} \cup F^{+}$, where $F^{-}=$ $F \cap\{(x, y, z): x<0\}$. For the rest of the proof, let $\mu=|F|$. If $H$ is an open half-space bounded by a plane containing the $x$-axis, by rotational symmetry, the Gauss images $\nu(M \cap H)=\mathrm{S}^{2} \cap H$. Consider various cases of convex $F$ in $\mathbf{S}^{2}$ depending on the area.

If $\mu_{1}=\pi(2-\sqrt{3})<\mu(\leq 2 \pi)$, there is some half-space $H$ bounded by a plane through the $x$-axis so that one of $\left\{H \cap F^{ \pm}, F^{ \pm}-H\right\}$ is null. Hence $I(F) \leq 3 \pi=2 \pi+J\left(\mu_{1}\right)<2 \pi+J(\mu)$.

In case $\mu \leq \mu_{2}=\pi(2-\sqrt{2})$, writing the convex set $F=F^{+} \cup F^{-}$ we claim if (29) holds for $F^{-}$, then it also holds for $F$. Using (29) and $\int_{R_{i}\left(f^{+}\right)} \kappa_{i} d a_{i}=\mu^{+}=\left|F^{+}\right|$we conclude

$$
2 \pi+J\left(\mu^{+}+\mu^{-}\right)-\int_{R_{i}\left(F^{+} \cup F^{-}\right)} \kappa_{i} d a_{i}>J\left(\mu^{+}+\mu^{-}\right)-\mu^{+}-J\left(\mu^{-}\right) .
$$

Under the hypothesis $\mu \leq \mu_{2}$ the right side of (30) is nonnegative, proving the claim.

Now if $0<\mu_{3} \leq \mu \leq \mu_{2}$, then $I\left(F^{-}\right) \leq 2 \pi<2 \pi+J\left(\mu_{3}\right) \leq 2 \pi+J(\mu)$. 
Finally suppose $\mu \leq \mu_{4}=\pi / 64$. By Lemma $6, F^{-}$is contained in a lune $V_{\pi / 2}$. The support of the curvature in $\left(\mathbf{S}^{2}\right)^{-}$is contained in a band of width $\cot ^{-1} i$ about the $x=0$ great circle. If the axis of the lune coincides with the $x$-axis, then $I\left(F^{-}\right) \leq I\left(V_{\pi / 2}^{-}\right) \leq \pi / 2$. Otherwise, let $P$ be a plane containing the $x$-axis and vertices of $V_{\pi / 2}$. If $V_{\pi / 2}$ is on one side of $P$, then $I\left(F^{-}\right) \leq I\left(V_{\pi / 2}^{-}\right) \leq \pi$, because $P$ cuts the band in half. If not, since the lune is on both sides of $P$, one of the faces of the lune makes an angle of at most $\pi / 4$ with $P$, thus avoiding the band of support at distances from $P$ greater than $\pi / 4$ on this side of $P$. In particular, if $i \geq 2$, the band width is small enough so that $\pi / 4$ of the band remains untouched by the lune. Hence $I\left(F^{-}\right) \leq I\left(V_{\pi / 2}^{-}\right) \leq \frac{7}{4} \pi<2 \pi$. Since each of the cases overlaps if $\mu_{3}<\mu_{4}$, we may extract a $\beta$ for (29).

\section{References}

[1] A. D. Alexandrov, On the theory of mixed volumes, Mat. Sb. 44 (1937) 947-972, 12051238; 45 (1938) 27-46, 227-251; English transl., Theory of mixed volumes, Oregon State University mimeograph, 1966.

[2] __ An application of the theorem of invariance of domains to existence proofs, Izv. Akad. Nauk SSSR 35 (1939) 243-256. (Russian)

[3] I. Bakelman \& B. Kantor, Existence of spherically homeomorphic hypersurfaces in Euclidean space with prescribed mean curvature, Geometry and Topology (Leningrad) 1 (1974), 3-10. (Russian)

[4] H. Busemann, Convex surfaces, Interscience, New York, 1958.

[5] L. Caffarelli, L. Nirenberg \& J. Spruck, Nonlinear second-order elliptic equations IV. Starshaped compact Weingarten hypersurfaces, Current Topics and Partial Differential Equations (Y. Ohya, K. Kasahara, N. Shimakura, eds.), Kinokunize, Tokyo, 1986, 1-26.

[6] P. Delanoë, Plongements $\mathbf{S}^{n} \rightarrow \mathbf{R}^{n+1}$ à courbure de Gauss positive prescrite, Ann. Sci. École Norm. Sup. (4) 18 (1985) 635-649.

[7] H. Federer, Geometric measure theory, Springer, Heidelberg, 1969.

[8] E. Guisti, Boundary value problems for non-parametric surfaces of prescribed mean curvature, Ann. Scuola Norm. Sup. Pisa Cl. Sci. (4) 3 (1976) 501-548.

[9] V. Oliker, Existence and uniqueness of convex hypersurfaces with prescribed Gaussian curvature in spaces of constant curvature, Sem. Inst. Mate. Appl. "Giovanni Sansone", Univ. Studi Firenze, 1983.

[10] _ Hypersurfaces in $R^{n+1}$ with prescribed Gaussian curvature and related equations of Monge Ampère type, Comm. Partial Differential Equations 9 (1984) 807-838.

[11] __ Three problems in geometry, Nonlinear Problems in Geometry, Proc. Conf. Mobile 1985 (D. DeTurck, ed.), Contemporary Math. vol. 51, Amer. Math. Soc. Providence, RI, 1986, 105-111.

[12] _ The problem of embedding $\mathbf{S}^{n}$ into $\mathbf{R}^{n+1}$ with prescribed Gauss curvature and its solution by variational methods, Trans. Amer. Math. Soc. 295 (1986) 291-303.

[13] A. V. Pogorelov, Extrinsic geometry of convex surfaces, "Nauka", Moscow, 1969; English transl., Transl. Math. Mono., Vol. 35, Amer. Math. Soc., Providence, RI, 1973. 
[14] L. Santalo, Integral geometry and geometric probability, Encyclopedia of Math. and its Application, Addison-Wesley, Reading, MA, 1976.

[15] A. Treibergs, Existence and convexity of hyperspheres of prescribed mean curvature, Ann. Scuola Norm. Sup. Pisa Cl. Sci. (4) 12 (1985) 225-241.

[16] A. Treibergs \& S. W. Wei, Embedded hyperspheres of prescribed mean curvature, J. Differential Geometry 18 (1983) 513-521.

\section{UNIVERSITY OF UTAH}

